\title{
Extent of Hypertension and Renal Injury in Children Surviving Acute Lymphoblastic Leukemia
}

\author{
Reeti Kumar ${ }^{1}$, John Mahan ${ }^{2}$, Joseph Stanek ${ }^{3}$, and Suzanne Reed ${ }^{2}$ \\ ${ }^{1}$ Duke University \\ ${ }^{2}$ Nationwide Children's Hospital \\ ${ }^{3}$ Nationwide Childrens Hospital
}

November 15, 2021

\begin{abstract}
Background: Childhood acute lymphoblastic leukemia (ALL) fortunately has high survival rates, and understanding longer-term implications of therapy is critical. In this study, we aimed to investigate kidney health outcomes by assessing the prevalence of renal dysfunction and hypertension (HTN) in children with ALL at one to five years after ALL diagnosis. Methods: This was a single center, cross-sectional study of children with ALL who were 1-5 years post diagnosis. GFR measurements were calculated, and urine samples were collected to assess for protein/creatinine and albumin/creatinine. Blood pressure (BP) was determined by standard oscillometric technique, and children $>6$ years of age were eligible for ambulatory blood pressure monitoring (ABPM). Results: Forty-five patients enrolled in the study, 21 completed ABPMs. Fifteen patients $(33 \%$, 95\% CI: 20-49\%) developed AKI at least once. Thirteen (29\%, 95\% CI: 16-44\%) had hyperfiltration, and 11 (24\%) had abnormal proteinuria and/or albuminuria. Prevalence of HTN based on clinic measurements was $42 \%$. In the 21 ABPM patients, 14 had abnormal results $(67 \%, 95 \%$ CI: 43-85\%) with the majority (11/21) demonstrating abnormal nocturnal dipping pattern. . Conclusions: Among children with ALL, there is a high prevalence of past AKI. The presence of hyperfiltration, proteinuria and/or albuminuria at 1-5 years after ALL diagnosis suggest real risk of developing CKD over time. There is a high prevalence of HTN on casual BP readings and even higher prevalence of abnormal ABPM in this group. The high prevalence of impaired nocturnal dipping by ABPM indicates an increased risk for future cardiovascular or cerebral ischemic events.
\end{abstract}

\section{Extent of Hypertension and Renal Injury in Children Surviving Acute Lymphoblastic Leukemia}

Reeti Kumar, MD ${ }^{1}$, John D. Mahan, $\mathrm{MD}^{2}$, Joseph R. Stanek, MS ${ }^{3}$, Suzanne Reed, MD

\section{Affiliations :}

${ }^{1}$ Department of Pediatrics, Division of Nephrology Duke University Medical Center Durham, NC 27705 USA

${ }^{2}$ Department of Pediatrics, Division of Nephrology Nationwide Children's Hospital Columbus, Ohio 432015 USA

${ }^{3}$ Department of Pediatrics, Division of Hematology/Oncology/Bone Marrow Transplant Nationwide Children's Hospital Columbus, Ohio 432015 USA

\section{Correspondence to:}

Suzanne Reed, MD

700 Children's Drive

Columbus $\mathrm{OH}$ 
43205

Phone: 614-722-6349

Fax: 614-722-1753

Suzanne.reed@nationwidechildrens.org

\section{Word counts}

Abstract: 250

Manuscript: 3230

\# of Tables: 1

\# of Figures: 1

Short title: Hypertension and Renal Injury in Survivors of ALL

List of key words : Acute kidney injury, chronic kidney disease, hypertension, Acute lymphoblastic leukemia

\section{Abbreviations:}

\begin{tabular}{ll}
\hline ABPM & ambulatory blood pressure monitoring \\
\hline ALL & acute lymphoblastic leukemia \\
BMT & bone marrow transplant \\
BP & blood pressure \\
CKD & chronic kidney disease \\
COG & Children's Oncology Group \\
DBP & diastolic blood pressure \\
eGFR & estimated glomerular filtration rate \\
GFR & glomerular filtration rate \\
HTN & hypertension \\
KDIGO & Kidney Disease: Improving Global Outcomes \\
MAP & mean arterial pressure \\
SBP & systolic blood pressure \\
TLS & tumor lysis syndrome \\
\hline
\end{tabular}

\section{Abstract}

Background:

Childhood acute lymphoblastic leukemia (ALL) fortunately has high survival rates, and understanding longer-term implications of therapy is critical. In this study, we aimed to investigate kidney health outcomes by assessing the prevalence of renal dysfunction and hypertension (HTN) in children with ALL at one to five years after ALL diagnosis.

\section{Methods:}

This was a single center, cross-sectional study of children with ALL who were 1-5 years post diagnosis. GFR measurements were calculated, and urine samples were collected to assess for protein/creatinine and albumin/creatinine. Blood pressure (BP) was determined by standard oscillometric technique, and children $>6$ years of age were eligible for ambulatory blood pressure monitoring (ABPM).

\section{Results:}


Forty-five patients enrolled in the study, 21 completed ABPMs. Fifteen patients (33\%, 95\% CI: 20-49\%) developed AKI at least once. Thirteen (29\%, 95\% CI: 16-44\%) had hyperfiltration, and 11 (24\%) had abnormal proteinuria and/or albuminuria. Prevalence of HTN based on clinic measurements was $42 \%$. In the 21 ABPM patients, 14 had abnormal results (67\%, 95\% CI: 43-85\%) with the majority (11/21) demonstrating abnormal nocturnal dipping pattern. .

\section{Conclusions :}

Among children with ALL, there is a high prevalence of past AKI. The presence of hyperfiltration, proteinuria and/or albuminuria at 1-5 years after ALL diagnosis suggest real risk of developing CKD over time. There is a high prevalence of HTN on casual BP readings and even higher prevalence of abnormal ABPM in this group. The high prevalence of impaired nocturnal dipping by ABPM indicates an increased risk for future cardiovascular or cerebral ischemic events.

\section{INTRODUCTION}

Acute lymphoblastic leukemia (ALL) is the most common pediatric cancer with an annual incidence of 30 cases per million persons younger than 20 in the United States. ${ }^{1,2}$ The cure rate of ALL has improved from less than $10 \%$ in the 1960's to around $90 \%$ in recent decades with innovations in chemotherapy, bone marrow transplantation $(\mathrm{BMT})$, and better supportive care ${ }^{1,3}$. However, with improvement in cure rate, there has been increased emergence of long-term therapy related complications such as musculoskeletal morbidity, cardiac comorbidities (congestive heart failure, coronary artery disease) and neurological comorbidities (cranial nerve palsy, cognitive defects) ${ }^{3}$. While there are several studies that address the prevalence of renal dysfunction and hypertension in children diagnosed and successfully treated for ALL, most of these studies investigated subjects with onset of disease prior to $2010 .{ }^{4-15}$

Children diagnosed with ALL are at risk for renal injury due to a number of factors including 1) intrinsic renal injury, secondary to either leukemic cell lysozyme induced direct tubular damage, leukemic infiltration, tumor lysis syndrome, sepsis, and/or exposure to nephrotoxic medications, 2) prerenal acute kidney injury from volume depletion, secondary to poor oral intake, vomiting and diarrhea, and 3) thrombotic microangiopathies. 4,16 Studies from prior decades reported that 10-30\% of survivors of childhood ALL have persistent reduced glomerular filtration rates (GFR). $., 6,7$

To more thoroughly understand renal injury, beyond assessing kidney function (GFR), presence of persistent proteinuria and albuminuria can indicate glomerular and/or tubular injury, and persistent proteinuria has been well documented as a major risk factor for the progression of renal disease ${ }^{17}$. To our knowledge, urine protein/creatinine and urine microalbumin/creatinine have not been previously reported in children with a history of ALL.

Hypertension (HTN), cardiovascular disease, and metabolic syndrome are known complications in patients who are long-term survivors of ALL. ${ }^{9,12,18,19}$ This has been attributed to multiple factors, including renal leukemic infiltration, persistent reduced glomerular filtration rate (GFR), chemotherapy, fluid overload, and exposure to chronic increased sodium loads from intravenous fluids and especially high dose steroids. ${ }^{10,11,20}$ Prior studies from the 1990's and 2000's show wide variation in incidence of HTN in children with ALL ranging from 5-65\%, depending on timing of blood pressure (BP) measurement and definition of HTN. 5,10,15,20,21 The latest clinical practice guideline for screening and management of high blood pressure in children and adolescents recommends obtaining a 24-hour ambulatory BP monitoring (ABPM) study in all children prior to establishing the diagnosis of hypertension. ${ }^{22}$ ABPM studies can help uncover "white coat HTN" (related to anxiety in clinical settings), masked HTN (normal office BP readings with elevated 24 hour mean BP), nocturnal HTN and abnormal nocturnal BP dipping (incomplete or absent decrease in BP while sleeping compared to while awake). To date, there has been only one study using ABPM in pediatric patients with ALL to assess for $\mathrm{HTN}^{23}$.

The aim of this study was to determine the prevalence of 1) persistent renal dysfunction based on assessments of GFR, proteinuria, and microalbuminuria and 2) HTN using in-clinic BP values and ABPM in children 
with ALL, at one to five years after ALL diagnosis.

\section{METHODS}

\section{Study Population}

Our study was a single center cross-sectional study conducted at Nationwide Children's Hospital from July 1, 2018 to February 28, 2019. All patients with ALL between the ages of 2-21 years who were 1-5 years post ALL diagnoses were eligible. There were no exclusion criteria. Subjects received chemotherapy according to various Children's Oncology Group (COG) protocols, including AALL0031, AALL0434, AALL0932, AALL1131, and AALL1231. The Institutional Review Board at Nationwide Children's Hospital approved this research study.

\section{Data Collection}

Demographic and clinical data were collected for each subject, including age at diagnosis, gender, ALL risk category, number of nephrotoxic agents received prior, number of hospitalizations, need for vasopressors, need for radiation therapy, obesity, current steroid use, history of AKI, and echocardiograms. Urine samples were collected at the time of study enrollment to assess for protein to creatinine and albumin to creatinine ratios. Serum creatinine obtained as standard of care at multiple times throughout ALL treatment was recorded from time of ALL diagnosis and then at three-month intervals up to time of study enrollment. Serum creatinine was measured using enzymatic method. Estimated glomerular filtration rate (eGFR) was calculated using bedside Schwartz and CKD-EPI creatinine equations 2009, depending on age of the subject ${ }^{24,25}$.

$\mathrm{BP}$ was measured by standard oscillometric methods at each clinic visit. Children 6 years and older were eligible for measurement of BP by ABPM. If they consented/assented to ABPM, a manual BP was obtained in the upper extremity and the appropriate size ABPM cuff and monitor were placed. The ABPM automatically measured BP values (systolic blood pressure (SBP), diastolic blood pressure (DBP) and mean arterial blood pressure (MAP)) every 20-30 minutes for a 24-hour period. If the subject was less than 6 years old or declined to wear an ABPM, a manual $\mathrm{BP}$ was obtained in clinic. If the $\mathrm{BP}$ was $>90^{\text {th }}$ percentile two repeat BP measuring were obtained at separate follow-up visits.

\section{Definitions}

AKI was defined according to the Kidney Disease: Improving Global Outcomes (KDIGO), modified to include only serum creatinine ${ }^{26}$. CKD was defined as GFR $<90 \mathrm{ml} / \mathrm{min} / 1.73 \mathrm{~m}^{2}$, CKD stage 2 as GFR $60-$ $89 \mathrm{ml} / \mathrm{min} / 1.73 \mathrm{~m}^{2}$, CKD stage 3 as GFR $30-59 \mathrm{ml} / \mathrm{min} / 1.73 \mathrm{~m}^{2}$, CKD stage 4 as GFR $15-29 \mathrm{ml} / \mathrm{min} / 1.73 \mathrm{~m}^{2}$, and CKD stage 5 (end stage renal disease) as GFR $<15 \mathrm{ml} / \mathrm{min} / 1.73 \mathrm{~m}^{2}$ for greater than 3 months respectively ${ }^{27}$. Renal hyperfiltration was defined as GFR $>2$ SD above mean GFR for age and gender ${ }^{28,29}$. Proteinuria was defined as urine protein/creatinine [?]0.2 $\mathrm{mg} / \mathrm{mg}$ and albuminuria as urine microalbu$\mathrm{min} /$ creatinine $>30 \mathrm{mg} / \mathrm{g}$.

Based on clinic BP measurements, all resting BP values were averaged and compared to age, sex, and height normal: ${ }^{22}$ normal BP was defined as BP $<90$ th percentile, elevated BP as BP [?] 90th percentile to $<95$ th percentile, stage 1 hypertension as $\mathrm{BP}$ [?] 95th percentile to $<95$ th percentile $+12 \mathrm{mmHg}$, and stage 2 hypertension as BP [?] 95th percentile $+12 \mathrm{mmHg}$.

In patients who completed ABPM, HTN was defined as either mean SBP and/or mean DBP [?] $95^{\text {th }}$ percentile for age, gender, and height. ${ }^{30}$ Night-day mean arterial pressure (MAP) ratios were calculated and dipping pattern was defined as follows: $<0 \%$ reverse dippers, [?]0 and $<10 \%$ non-dippers, [?]10 and $<20 \%$ normal dippers, and [?] $20 \%$ extreme dippers. ${ }^{31}$

ALL risk groups were defined using the subject's treatment protocol, which is assigned early in treatment, based on a combination of several factors: age at diagnosis, presenting white blood count, cytogenetics, and response to therapy. Tumor lysis syndrome (TLS) was defined per Cairo-Bishop definition of laboratory TLS. $^{32}$ 


\section{Statistical analysis}

All data were summarized using standard descriptive statistical methods. Prevalence values were reported with corresponding exact binomial 95\% confidence intervals (CI). Comparisons of qualitative variables between patient groups were completed using chi-square tests, and comparisons between quantitative variables between patient groups were completed using either t-tests or, in the case of non-normality, Mann-Whitney $\mathrm{U}$ tests. The comparison of SBP and DBP indices at diagnosis and 1-month post-diagnosis was completed using paired t-tests. Logistic regression models were used to assess risk factors for the occurrence of AKI, CKD, and HTN. Variables assessed included age at diagnosis, gender, ALL risk category, number of nephrotoxic agents, number of hospitalizations, need for vasopressors, need for radiation, obesity, current steroid use, history of AKI. All analyses were performed using SAS software, version 9.4 (SAS Institute, Cary, NC).

\section{RESULTS}

\section{Patient Characteristics}

A total of forty-five patients enrolled in the study, of which twenty-one completed ABPMs. Baseline demographic data and specific clinical characteristics are presented in Table 1. Demographics included predominance of males (71\%), Caucasians (96\%), B-cell ALL (80\%), and high-risk ALL (62\%). The median age at time of diagnosis was 4 years and median age at time of study visit was 7 years. Four patients had pre-existing renal disease prior to diagnosis of ALL. Twenty-nine patients were receiving active ALL therapy at time of enrollment in the study, and eighteen patients were receiving steroids at enrollment.

\section{Renal Dysfunction}

The overall prevalence of a history of AKI in our cohort was high, with 15 subjects (33\%, 95\% CI: 20-49\%) developing AKI at least once. The majority of AKI occurred at time of diagnosis $(10 / 15,66.7 \%)$. Of the 15 subjects with AKI, 13 (86.7\%) had KDIGO stage 1 AKI, 1 (6.7\%) had KDIGO stage 2 AKI, and $1(6.7 \%)$ had KDIGO stage 3 AKI. In the 28 high-risk ALL patients, 13 had AKI (46\%), while in the 17 standard-risk ALL patients, only 2 had AKI (11.8\%). Those treated with high-risk ALL therapy were more likely to have AKI than those treated with standard-risk ALL therapy $(\mathrm{P}=0.017)$. In univariate analysis, predictors for AKI included increasing age at time of diagnosis (median age with AKI 9.5yr vs median age with no AKI 4.3yr, $\mathrm{P}=0.006)$ and high-risk ALL $(\mathrm{P}=0.026)$. Interestingly, number of nephrotoxic agents, number hospitalizations, and need for vasopressors were not found to be significant risk factors for AKI.

No patients in this short period of follow-up demonstrated CKD. However, 13 of 45 patients $(28.9 \%, 95 \%$ CI: 16.4-44.3\%) had hyperfiltration, which represents a supraphysiologic elevation in GFR. Last recorded GFR for patients are shown in figure 1 . The mean last GFR was $146.1 \mathrm{ml} / \mathrm{min} / 1.73 \mathrm{~m}^{2}$ (range $74-228$ $\mathrm{ml} / \mathrm{min} / 1.73 \mathrm{~m}^{2}$ ). The median month that last GFR was calculated was 30 months (range 15-60 months) post diagnosis. In the 28 high-risk ALL patients, 9 had hyperfiltration (32.1\%). In the 17 standard-risk ALL patients, 4 had hyperfiltration (23.5\%). In light of the small sample size, no variable was identified as a risk factor for developing hyperfiltration.

Urinalysis was completed in 41 patients, as 4 were unable to provide a sample. Eleven of 41 patients (24\%) had abnormal urinalysis. One patient $(2 \%)$ had proteinuria alone, 6 patients (15\%) had albuminuria alone, and 3 patients $(7 \%)$ had combined proteinuria and albuminuria. Univariate analysis in the proteinuria group was not performed due to small sample size. Univariate analysis in the microalbuminuria group did not result in identification of any significant risk factors.

\section{HTN}

The prevalence of HTN in all patients was high, with 19 of 45 having HTN (42.2\%, 95\% CI: 22.0-63.6\%). In univariate analysis, no variable was significant as a risk factor for HTN. In these 19 HTN patients, one had left ventricular hypertrophy noted on previous echocardiogram. None of the 26 non-HTN patients had LVH on previous echocardiograms. However, these echocardiograms were obtained as part of standard of care prior to initiation of ALL therapy rather than at time of enrollment in this renal outcomes study. Eight of 
24 patients were on chronic anti-HTN treatment at some point in time and 2 of these patients were actively taking anti-HTN medications at the time of study enrollment. All patient who received chronic anti-HTN treatment were prescribed the calcium channel blocker amlodipine.

In the 21 patients who completed an ABPM study, 14 had an abnormal result (67\%, 95\% CI: 43-85\%). Of these 21 patients, 13 had normal casual BP values and of this group, 7 (54\%) had abnormal ABPM readings, therefore being designated as having abnormal BP by ABPM even though by casual BP readings their BP was normal. On ABPM, one patient with known HTN had well-controlled BP on chronic anti-HTN medication. One patient was noted to have pre-HTN. One patient was noted to have nocturnal HTN. Eleven patients had abnormal nocturnal dipping pattern: 10 patients were non-dippers, with no physiologic decline in sleep BPs, and 1 patient was an extreme dipper, with greater than $20 \%$ decline in sleep MAP compared to wake MAP. In univariate analysis, no variable was significant as a risk factor for abnormal ABPM.

\section{DISCUSSION}

In this cross-sectional study, we found a high incidence of AKI in children diagnosed with ALL, with $33 \%$ of subjects having at least one AKI episode and the majority of AKI episodes noted at time of ALL diagnosis. Most patients had mild AKI (KDIGO stage 1). Patients with high-risk ALL had a higher prevalence of AKI compared to patients with standard-risk ALL. This may have been due to greater likelihood of leukemic infiltration in the kidneys at diagnosis, greater risk of tumor lysis syndrome with initial chemotherapy, and/or the more toxic chemotherapy regimens used in high-risk ALL which can be directly nephrotoxic and increase risk for complications such as sepsis.

No patients were found to have CKD in this early follow-up study, a better short-term outcome than that reported by studies from previous decades, and perhaps a reflection of better supportive care now available for children with ALL. ${ }^{4,6,7}$ Almost a third of patients were noted to have hyperfiltration, however, and in other populations this identifies increased risk for subsequent renal injury and progressive CKD. ${ }^{33,34}$ The mean last recorded GFR of the overall group was supraphysiologic, worrisome for potential future renal complications. Additionally, $24 \%$ of patients who completed a urinalysis were noted to have either proteinuria, albuminuria or both.

The low prevalence of CKD in our cohort is likely related to short duration of follow-up time. In a recent Israeli study evaluating the risk of ESRD in childhood cancer survivors with median follow-up time of 30 years, participants with history of malignancy in childhood had adjusted HR of 3.2 of developing ESRD compared to participants without no history of tumors/malignancy in childhood. ${ }^{35}$ In the 1990 's, KrawczukRybak et al. found 5/37 (13.5\%) childhood ALL survivors had abnormal creatinine clearance when followed $3.9 \pm 3.7$ years after completion of chemotherapy ${ }^{6}$. Yetgin et al. detected reduced GFR in 22/116 (19\%) of pediatric ALL patients treated mostly in the 1990's who were assessed 18-96 months after completion of chemotherapy ${ }^{4}$. Yetgin also performed urinalysis on all subjects post chemotherapy and found no evidence of proteinuria in these patients. However, 7/116 (6\%) of patients had high urine $\mathrm{B}_{2}$-Microglobulin, a marker of renal tubular proteinuria. Hovi et al. reported GFR $<85 \mathrm{ml} / \mathrm{min} / 1.73 \mathrm{~m}^{2}$ in $6 / 60(10 \%)$ of children with ALL followed 1-9 years after completion of chemotherapy, but this study presents data from over 25 years ago. $^{7}$

The high prevalence of hyperfiltration, proteinuria, and/or albuminuria noted in our cohort suggests that these children with ALL, treated since 2015, are at risk of developing CKD in the long term. This phenomenon is well-described in patients with diabetic nephropathy. Glomerular hyperfiltration commonly occurs early in diabetes secondary to increased glomerular capillary pressure. Over time, sustained elevated GFR can result in mesangial and podocyte injury and eventually glomerular scarring leading to CKD progression and ESRD. ${ }^{33,34,36}$ The presence of albuminuria/proteinuria in patients with diabetes or CKD has been strongly associated with increased risk of subsequent renal function decline and measures that are successful at reducing albuminuria/proteinuria have been shown to slow the renal disease progression in these populations. ${ }^{36,37}$

Our study also found that children with ALL have a high prevalence of HTN. 
Almost half of our study patients (42\%) fulfilled criteria for outpatient diagnosis of HTN based on clinic BP measurements, termed casual BP readings. Our findings are consistent with prior ALL survivorship research, which has reported incidences of HTN in children with ALL ranging from 5-65\%, with variations likely affected by study methodology and HTN definitions. ${ }^{5,10,15,20,21}$ The high incidence of HTN is likely multifactorial, related to past or present corticosteroid therapy, chemotherapy, prior AKI, and/or renal leukemic infiltration. Prior studies have also proposed metabolic syndrome and obesity as risk factors for developing chronic HTN in adults who survive childhood leukemia. ${ }^{5,8,9,12-14}$

In the 21 patients who completed an ABPM study, we found that the percentage of individuals with HTN was much higher, since $67 \%$ had an abnormal high BP value. The majority of patients with abnormal ABPM had abnormal nocturnal dipping pattern. Ociepa et al. is the only other study to report ABPM results as a method to diagnose HTN in children with ALL. This study detected a higher prevalence of childhood survivors of ALL with HTN via ABPM $(37 \%, 30 / 81)$, although it is perhaps noteworthy that their study had a longer mean time of follow-up (60 months) compared to our study (36 months). Similar to our study, Ociepa et al. found that childhood survivors of ALL were more likely to have abnormal nocturnal dipping pattern (nondippers, reverse-dippers, and extreme-dippers) compared to control population. In healthy children during sleep, a 10-20\% physiologic decrease of BP is normally noted. The lack of this physiologic decrease is termed non-dipping and BP decrease in excess of $20 \%$ is considered extreme dipping. Both non-dipping and extreme dipping have been associated with increased risk of cardiovascular or cerebral ischemic/hemorrhagic events in adults. ${ }^{38-42}$ In pediatric subjects with systemic lupus erythematosus, non-dipping has been associated with endothelial dysfunction and atherosclerotic changes. ${ }^{43}$ Similarly, in adolescents with diabetes, nondipping pattern has been associated with LVH and increased carotid intimal media thickness ${ }^{44,45}$. Further studies of these ABPM abnormalities and outcomes in children with ALL will help understand the prognostic implications and identify opportunities for useful interventions in these children.

Limitations of this study include its single-center design with relatively small sample size and short duration of follow-up. A multi-center study with larger sample size could usefully confirm the findings of this study and help determine distinct risk factors for developing renal dysfunction and hypertension. It should also be noted in this study that urine samples were collected at the time of clinic appointment for convenience and were not first morning samples. Thus, orthostatic proteinuria (a benign phenomenon occurring in up to $7 \%$ of children and adolescents) could account for some of the proteinuria/albuminuria noted in our findings ${ }^{46}$.

In conclusion, our study showed that, among children with ALL, there is a high prevalence of HTN based on casual BP readings obtained in the clinic setting and a high prevalence of impaired nocturnal dipping based on ABPM. Both abnormalities are associated with increased risk of cardiovascular disease and cerebrovascular events later in life. While the prevalence of CKD is very low, children with ALL have a high prevalence of hyperfiltration and/or proteinuria/albuminuria suggesting increased risk of developing CKD in the future.

Interestingly, screening urinalysis has been removed from the Children's Oncology Group long-term follow-up guidelines for children who survive ALL. ${ }^{47}$ The value of a screening urinalysis in detection of early evidence of renal disease is highlighted by the Prevention of Renal and Vascular End-Stage Disease (PREVEND) study conducted of over 40,000 adult participants which indicated the usefulness of albuminuria in early detection of CKD. PREVEND found that of individuals who required renal replacement therapy after 9-years followup, $58 \%$ had moderately increased albuminuria ([?]20mg/L) on early screening urine sample. Increased albuminuria was an independent risk factor for progression to ESRD independent of GFR. ${ }^{48}$ Based on our findings and other recent studies, ${ }^{3,7,20}$ we propose that children with ALL should undergo regular (yearly) renal function testing, urinalysis and BP measurements to screen for early signs of renal abnormalities. Longer term and larger studies will be needed to confirm our findings and define predictors for these adverse outcomes in children with ALL.

As the long-term cure rate of ALL has improved to greater than 90\%, the challenge now extends to achieving the same cure rates with reduced short-term and long-term toxicities for these children. One treatment modality which shows promise in this goal is immunologic therapy. There have been several recent trials demonstrating the safety and efficacy of antibody-based and T cell-based therapies for ALL. ${ }^{49,50}$ Our find- 
ings demonstrate that current conventional therapies for childhood ALL are accompanied by concerning frequencies of early renal dysfunction and hypertension. The shift towards targeted immunologic therapies may mitigate some of these long-term renal consequences of conventional therapy and provide better quality of life in survivors of childhood ALL.

Conflicts of Interest Statement: The authors have no conflicts of interest

Acknowledgements: This work was supported by an intramural grant from Nationwide Children's Hospital

\section{References}

1. Hunger SP, Mullighan CG. Acute Lymphoblastic Leukemia in Children.New England Journal of Medicine 2015; 373 (16): 1541-52.

2. Siegel RL, Miller KD, Jemal A. Cancer statistics, 2017. CA: A Cancer Journal for Clinicians 2017; 67 (1): 7-30.

3. Mody R, Li S, Dover DC, et al. Twenty-five-year follow-up among survivors of childhood acute lymphoblastic leukemia: a report from the Childhood Cancer Survivor Study. Blood 2008; 111 (12): 5515-23.

4. Yetgin S, Olgar S, Aras T, et al. Evaluation of kidney damage in patients with acute lymphoblastic leukemia in long-term follow-up: Value of renal scan. American Journal of Hematology 2004;77 (2): 132-

5. Chow EJ, Pihoker C, Hunt K, Wilkinson K, Friedman DL. Obesity and hypertension among children after treatment for acute lymphoblastic leukemia. Cancer 2007; 110 (10): 2313-20.

6. Krawczuk-Rybak MM. Renal function during and after treatment for acute lymphoblastic leukemia in children. Pediatric nephrology (Berlin, West) 2005; 20 (6): 782-5.

7. Hovi L. Risk of progressive kidney damage after acute leukemia.Acta pædiatrica Scandinavica 1989; 78 (4): 608-14.

8. Veringa SJE, van Dulmen-den Broeder E, Kaspers GJL, Veening MA. Blood pressure and body composition in long-term survivors of childhood acute lymphoblastic leukemia. Pediatric Blood \& Cancer 2012;58 (2): $278-82$.

9. Levy E, Samoilenko M, Morel S, et al. Cardiometabolic Risk Factors in Childhood, Adolescent and Young Adult Survivors of Acute Lymphoblastic Leukemia - A Petale Cohort. Scientific Reports 2017;7 (1): 17684.

10. Louis CUCU. High blood pressure and hypertension in children with newly diagnosed acute leukemia and lymphoma. Pediatric nephrology (Berlin, West) 2008; 23 (4): 603-9.

11. Attard-Montalto SPS. High incidence of hypertension in children presenting with acute lymphoblastic leukemia. Pediatric hematology and oncology 1994; 11 (5): 519-25.

12. Nottage KA, Ness KK, Li C, Srivastava D, Robison LL, Hudson MM. Metabolic syndrome and cardiovascular risk among long-term survivors of acute lymphoblastic leukaemia - From the St. Jude Lifetime Cohort.British Journal of Haematology 2014; 165 (3): 364-74.

13. Saultier P, Auquier P, Bertrand Y, et al. Metabolic syndrome in long-term survivors of childhood acute leukemia treated without hematopoietic stem cell transplantation: an L.E.A. study.Haematologica 2016; 101 (12): 1603-10.

14. Oudin C, Simeoni M-C, Sirvent N, et al. Prevalence and risk factors of the metabolic syndrome in adult survivors of childhood leukemia.Blood 2011; 117 (17): 4442-8.

15. Bakk I, O'Brien S, Reed S, Koch T. Steroid-Induced Hypertension during Induction Chemotherapy for Acute Lymphoblastic Leukemia in US Children's Hospitals. Jour Pediatr Hematol Oncol. 2014;124 (21): 3651-. 
16. Luciano RL, Brewster UC. Kidney Involvement in Leukemia and Lymphoma. Advances in Chronic Kidney Disease 2014;21 (1): 27-35.

17. Bakris GLGL. Slowing nephropathy progression: focus on proteinuria reduction. Clinical journal of the American Society of Nephrology2008; 3 Suppl 1 (supplement 1): S3-S10.

18. Oeffinger KC. Cardiovascular risk factors in young adult survivors of childhood acute lymphoblastic leukemia. Journal of pediatric hematology/oncology 2001; 23 (7): 424-30.

19. Talvensaari KK, Lanning M, Tapanainen P, Knip M. Long-term survivors of childhood cancer have an increased risk of manifesting the metabolic syndrome. The Journal of Clinical Endocrinology ES Metabolism1996; 81 (8): 3051-5.

20. Olgar SS. Can renal leukemic infiltration cause hypertension in children? Journal of pediatric hematology/oncology 2006;28 (9): 579-84.

21. Essig S, Li Q, Chen Y, et al. Risk of late effects of treatment in children newly diagnosed with standardrisk acute lymphoblastic leukaemia: a report from the Childhood Cancer Survivor Study cohort. The Lancet Oncology 2014; 15 (8): 841-51.

22. Flynn JT, Kaelber DC, Baker-Smith CM, et al. Clinical Practice Guideline for Screening and Management of High Blood Pressure in Children and Adolescents. Pediatrics 2017.

23. Ociepa T, Bartnik M, Zielezinska K, Urasinski T. Prevalence and Risk Factors for Arterial Hypertension Development in Childhood Acute Lymphoblastic Leukemia Survivors. Journal of Pediatric Hematology/Oncology 2019; 41 (3): 175-80.

24. Levey AS, Stevens LA. Estimating GFR using the CKD Epidemiology Collaboration (CKD-EPI) creatinine equation: more accurate GFR estimates, lower CKD prevalence estimates, and better risk predictions. $A m$ J Kidney Dis 2010; 55 (4): 622-7.

25. Staples A, LeBlond R, Watkins S, Wong C, Brandt J. Validation of the revised Schwartz estimating equation in a predominantly non-CKD population. Pediatr Nephrol 2010; 25 (11): 2321-6.

26. Group KDIGOAKIW. KDIGO clinical practice guideline for acute kidney injury. Kidney Int Suppl 2012; 2 (1): 1-138.

27. Inker LA, Astor BC, Fox CH, et al. KDOQI US Commentary on the 2012 KDIGO Clinical Practice Guideline for the Evaluation and Management of CKD. American Journal of Kidney Diseases ; 63 (5): 713-35.

28. Hogg RJ, Furth S, Lemley KV, et al. National Kidney Foundation's Kidney Disease Outcomes Quality Initiative Clinical Practice Guidelines for Chronic Kidney Disease in Children and Adolescents: Evaluation, Classification, and Stratification. Pediatrics 2003;111 (6): 1416-21.

29. Altay S, Onat A, Özpamuk-Karadeniz F, Karadeniz Y, Kemaloğlu-Öz T, Can G. Renal "hyperfiltrators" are at elevated risk of death and chronic diseases. BMC nephrology 2014; 15 : 160-.

30. Flynn JT, Daniels SR, Hayman LL, et al. Update: Ambulatory Blood Pressure Monitoring in Children and Adolescents. A Scientific Statement From the American Heart Association 2014; 63 (5): 1116-35.

31. Urbina E, Alpert B, Flynn J, et al. Ambulatory Blood Pressure Monitoring in Children and Adolescents: Recommendations for Standard Assessment. A Scientific Statement From the American Heart Association Atherosclerosis, Hypertension, and Obesity in Youth Committee of the Council on Cardiovascular Disease in the Young and the Council for High Blood Pressure Research 2008; 52 (3): 433-51.

32. Mirrakhimov AE, Voore P, Khan M, Ali AM. Tumor lysis syndrome: A clinical review. World J Crit Care Med 2015; 4 (2): 130-8. 
33. Jerums G, Premaratne E, Panagiotopoulos S, MacIsaac RJ. The clinical significance of hyperfiltration in diabetes. Diabetologia 2010;53 (10): 2093-104.

34. Magee GM, Bilous RW, Cardwell CR, Hunter SJ, Kee F, Fogarty DG. Is hyperfiltration associated with the future risk of developing diabetic nephropathy? A meta-analysis. Diabetologia 2009; 52 (4): 691.

35. Calderon-Margalit R, Pleniceanu O, Tzur D, et al. Childhood Cancer and the Risk of ESKD. Journal of the American Society of Nephrology 2020: ASN.2020071002.

36. Mogensen CE, Christensen CK. Predicting Diabetic Nephropathy in Insulin-Dependent Patients. New England Journal of Medicine 1984;311 (2): 89-93.

37. Bakris GL. Slowing nephropathy progression: focus on proteinuria reduction. Clinical journal of the American Society of Nephrology : CJASN 2008; 3 Suppl 1 (Suppl 1): S3-S10.

38. Phillips RA, Sheinart KF, Godbold JH, Mahboob R, Tuhrim S. The association of blunted nocturnal blood pressure dip and stroke in a multiethnic population. Am J Hypertens 2000; 13 (12): 1250-5.

39. Fagard RH, Celis H, Thijs L, et al. Daytime and Nighttime Blood Pressure as Predictors of Death and Cause-Specific Cardiovascular Events in Hypertension. Hypertension 2008; 51 (1): 55-61.

40. Ben-Dov IZ, Kark JD, Ben-Ishay D, Mekler J, Ben-Arie L, Bursztyn M. Predictors of All-Cause Mortality in Clinical Ambulatory Monitoring. Hypertension 2007; 49 (6): 1235-41.

41. Boggia J, Li Y, Thijs L, et al. Prognostic accuracy of day versus night ambulatory blood pressure: a cohort study. The Lancet 2007;370 (9594): 1219-29.

42. Kario K, Pickering Thomas G, Matsuo T, Hoshide S, Schwartz Joseph E, Shimada K. Stroke Prognosis and Abnormal Nocturnal Blood Pressure Falls in Older Hypertensives. Hypertension 2001; 38 (4): 852-7.

43. Chang JC, Xiao R, Meyers KE, et al. Nocturnal blood pressure dipping as a marker of endothelial function and subclinical atherosclerosis in pediatric-onset systemic lupus erythematosus. Arthritis Research E Therapy 2020; 22 (1): 129.

44. Karavanaki K, Kazianis G, Konstantopoulos I, Tsouvalas E, Karayianni C. Early signs of left ventricular dysfunction in adolescents with type 1 diabetes mellitus: the importance of impaired circadian modulation of blood pressure and heart rate. J Endocrinol Invest 2008;31 (4): 289-96.

45. Lee SH, Kim JH, Kang MJ, Lee YA, Won Yang S, Shin CH. Implications of nocturnal hypertension in children and adolescents with type 1 diabetes. Diabetes Care 2011; 34 (10): 2180-5.

46. Brandt JR, Jacobs A, Raissy HH, et al. Orthostatic proteinuria and the spectrum of diurnal variability of urinary protein excretion in healthy children. Pediatric Nephrology 2010; 25 (6): 1131-7.

47. Group CsO. Long-Term Follow-up Guidelines for Survivors of Childhood, Adolescent and Young Adult Cancerns. Monrovia, CA.

48. van der Velde M, Halbesma N, de Charro FT, et al. Screening for Albuminuria Identifies Individuals at Increased Renal Risk. Journal of the American Society of Nephrology 2009;20 (4): 852-62.

49. Hoelzer D. Chemotherapy-free Treatment - A New Era in Acute Lymphoblastic Leukemia? New England Journal of Medicine 2020;383 (17): 1673-4.

50. Inaba H, Pui CH. Immunotherapy in pediatric acute lymphoblastic leukemia. Cancer Metastasis Rev 2019; 38 (4): 595-610.

\section{Hosted file}

PBC.RenOut.Tab.Fig.docx available at https://authorea.com/users/446271/articles/545565extent-of-hypertension-and-renal-injury-in-children-surviving-acute-lymphoblasticleukemia 